\title{
Automatización de un banco de ensayos de generadores eléctricos para aplicaciones en energía eólica de baja potencia
}

\author{
Automated electric generators test bench for low power wind energy applications
}

\author{
Juan Carlos Agotegaray ${ }^{1}$, Andrea Pinzón ${ }^{2}$, Emanuel Lera ${ }^{3}$ \\ Instituto de Industria, Universidad Nacional de General Sarmiento, Los Polvorines, Argentina \\ jjagotega@campus.ungs.edu.ar \\ 2apinzon@ campus.ungs.edu. ar \\ ${ }^{3}$ elerae campus.ungs.edu.ar \\ Recibido: 24/09/19; Aceptado: 04/01/20
}

\begin{abstract}
Resumen-En este trabajo se presenta el desarrollo de un banco de ensayos automatizado de generadores eléctricos, con aplicación en energía eólica de baja potencia y uso en zonas urbanas. La implementación de este banco permite ensayar generadores eléctricos bajo condiciones de funcionamiento semejantes a los que se sometería como generador acoplado a una turbina eólica en entornos urbanos, a partir de lo cual, es posible determinar los parámetros específicos: corriente, tensión, potencia, que permitan caracterizarlo.
\end{abstract}

Keywords: Ensayos, Automatización, Aerogenerador, Generador, Control

Abstract - This paper presents the development of an automated electric generators test bench for low power wind energy in urban applications. The bench allows testing electric generator under similar operating conditions to real, electric generator coupled to a wind turbine in urban environments, which allows to determine specific parameters: electric current, voltage, power.

Keywords: Testing, Automation, Wind Turbine, Generator, Control

\section{INTRODUCCION}

La generación eléctrica distribuida con paneles solares y aerogeneradores se encuentra cada vez más presente entre los usuarios particulares. Según datos de CAMMESA (Compañía Administradora del Mercado Eléctrico Mayorista), en Argentina al día de hoy la potencia instalada de generación eléctrica a partir de energías renovables alcanzaba el 8,6\% del total [1]. En general, cuando se habla de energía eólica se piensa en los sistemas de alta potencia, es decir, parques eólicos con potencia instalada en el orden de los MW (Megawatts), que puedan despachar su energía en el Sistema Argentino Interconectado (SADI). Sin embargo, existen zonas urbanas en los cuales se puede generar energía eléctrica a partir de energía eólica, en menor escala. Para implementar aerogeneradores en estas zonas, los mismos deben adaptarse a los vientos de baja velocidad que se dan debido a la irregularidad de los edificios, árboles y demás obstáculos. En estas condiciones son recomendables los aerogeneradores de eje vertical [2].
Existen distintos tipos de generadores eléctricos para ser usados en aerogeneradores según la aplicación: alta o baja potencia, conectado o aislado a la red. En la Universidad Nacional de General Sarmiento, en el marco del proyecto de investigación "Generadores Eólicos Urbanos" radicado en el Instituto de Industria, con financiamiento de la Secretaría de Políticas Universitarias (SPU), se viene realizando el análisis de las tecnologías existentes y de los proyectos desarrollados en el país para la generación eléctrica domiciliaria. Dentro del proyecto se está desarrollando un aerogenerador de eje vertical. La turbina eólica es de tipo Darrieus $\mathrm{H}$ y el generador eléctrico es una maquina síncrona trifásica de flujo axial. Estas dos máquinas se acoplan de forma directa, por lo que la frecuencia de la onda sinusoidal de tensión que se obtiene del generador depende directamente de la velocidad de rotación (RPM) de la turbina. Esta máquina fue diseñada y construida teniendo en cuenta los parámetros de funcionamiento de la turbina eólica. Para analizar el funcionamiento del generador eléctrico se realizaron mediciones eléctricas y mecánicas en un banco de ensayo.

Inicialmente las mediciones sobre el banco de ensayos se realizaban de forma manual. Luego, se planteó la necesidad de normalizar las mediciones implementando un PLC (Programmable Logic Controller) que pueda registrar datos $\mathrm{y}$ controlar el funcionamiento del motor que impulsa el generador eléctrico. Con esto, se logró caracterizar el generador eléctrico de flujo axial ya construido y disponer de una herramienta para realizar ensayos para el estudio de los futuros desarrollos.

Actualmente se está trabajando en la construcción de otro generador eléctrico tipo brushless, para bajas velocidades de viento, que se caracteriza por tener gran torque de arranque debido al número de polos magnéticos que tiene el estator, los cuales pueden reconfigurarse para obtener una tensión de generación en función de las RPM (revolución por minuto) de funcionamiento. Se espera de los ensayos del motor brushless, en un rango de velocidades entre 50 y 300 RPM (rango de funcionamiento definido para el aerogenerador), obtener una potencia aproximada de $100 \mathrm{~W}$ y almacenar la energía en un banco de baterías de 24 voltios. 


\section{AEROGENERADOR}

\section{A. Turbina Eólica Darrieus H}

Para transformar la energía cinética contenida en el viento se utiliza una turbina eólica, que para el caso, es de eje vertical, es decir, su eje de rotación es perpendicular a la dirección de viento. Este tipo de aerogeneradores tienen ventajas como la posibilidad de funcionar con vientos que provengan desde cualquier dirección y con cierto nivel de turbulencia. Este comportamiento se da normalmente en zonas urbanas, donde la rugosidad superficial hace que los vientos presenten características aleatorias. La turbina eólica Darrieus H está formada por palas de perfil normalmente biconvexo, unidas rígidamente entre sí, que giran alrededor de un eje vertical [4]. En el proyecto, la turbina fue construida con tres palas fabricadas a partir de un perfil NACA 0018 , con una cuerda de 200 milímetros y 1 metro de largo, definiendo un diámetro total de 1,1 metros, la misma se observa en la figura 1. El ángulo pitch $\beta$ que se forma entre la dirección de rotación y la cuerda de las palas es de $0^{\circ}$.

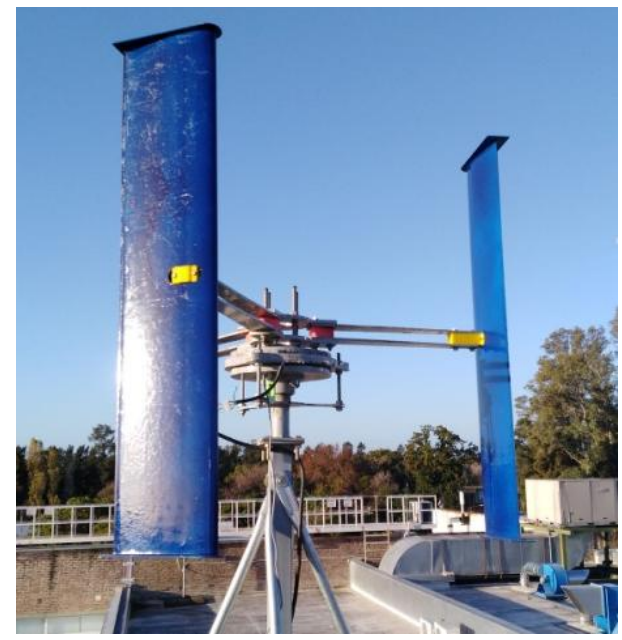

Fig 1. Aerogenerador Darrieus H.

\section{B. Generador Eléctrico de Flujo Axial}

Existen distintos tipos de generadores eléctricos que se acoplan a las turbinas eólicas para poder producir energía eléctrica. Los aerogeneradores de baja potencia se implementan normalmente en zonas aisladas de la red eléctrica como una fuente de generación, como los sistemas fotovoltaicos o los grupos electrógenos. Por esta razón, se recurren a generadores de diseño robustos y con mantenimiento mínimo, entre los distintos tipos, se destaca el generador sincrónico de flujo axial con imanes permanentes. Este tipo de generador posee rotores con polos magnéticos cuyas líneas de campo atraviesan axialmente un estator de bobinas donde se induce la corriente eléctrica. Tienen la particularidad de poder tener un gran número de polos magnéticos en poco espacio, lo que es ideal para aplicaciones de baja velocidad. En el marco del proyecto, se construyó un generador eléctrico de este tipo, tomando como base el diseño implementado en el aerogenerador de autoconstrucción Piggot [5]. El generador eléctrico tiene una configuración trifásica de 12 pares de polos distribuidos en dos rotores y 9 bobinas en un estator central.

\section{BANCO DE ENSAYOS}

El estudio del generador eléctrico se realizó con el fin de determinar su rendimiento, para lo cual se lo consideró, al generador y al sistema de rectificación, como una caja negra sobre la cual se realizan mediciones para conocer la energía mecánica que ingresa y la energía eléctrica que sale. El banco de ensayos debía permitir realizar mediciones eléctricas y mecánicas sobre el generador eléctrico mientras este fuera accionado de forma directa por un motor trifásico Siemens 1LE0102-0EC4 de 1,1 kW [6]. Con esto se buscó simular las condiciones de funcionamiento a distintas velocidades de rotación que le transmite la turbina eólica al generador. Para controlar el funcionamiento del motor trifásico, se empleó un variador de frecuencia Siemens V20 de 1,5 kW [7], el cual puede recibir las órdenes de funcionamiento a través de un panel de operador llamador por Siemens MOP (motorized potentiometer). El motor trifásico y el generador eléctrico se montaron en una estructura metálica especialmente construida para soportar y fijar ambos equipos, y mediante un acople fabricado en una impresora 3D se vincularon permitiendo la trasmisión de potencia mecánica.

En un principio, las mediciones eléctricas de tensión y corriente en continua, y mecánicas de RPM se realizaban con instrumentos manuales. Se configuraba el variador de frecuencia para que el motor gire a una determinada velocidad y se realizaban las mediciones.

\section{A. Tablero de control del banco de ensayos}

En el proyecto se decidió construir un tablero de control en el cual se instaló el variador de frecuencia y un PLC Siemens S7 200 CPU 222 [8] con un módulo EM 235 para procesar señales analógicas. Con este equipamiento se mejoró el sistema de medición del banco de ensayos al tener la posibilidad de registrar señales digitales y analógicas provenientes de los sensores instalados sobre el generador eléctrico a analizar, así como también, la posibilidad de controlar el funcionamiento del variador de frecuencia enviándole órdenes mediante señales digitales del PLC y la consigna de velocidad con una señal analógica. El esquema del sistema se presenta en la figura 2 y el banco de ensayos de y el tablero de control se observan en la figura 3.

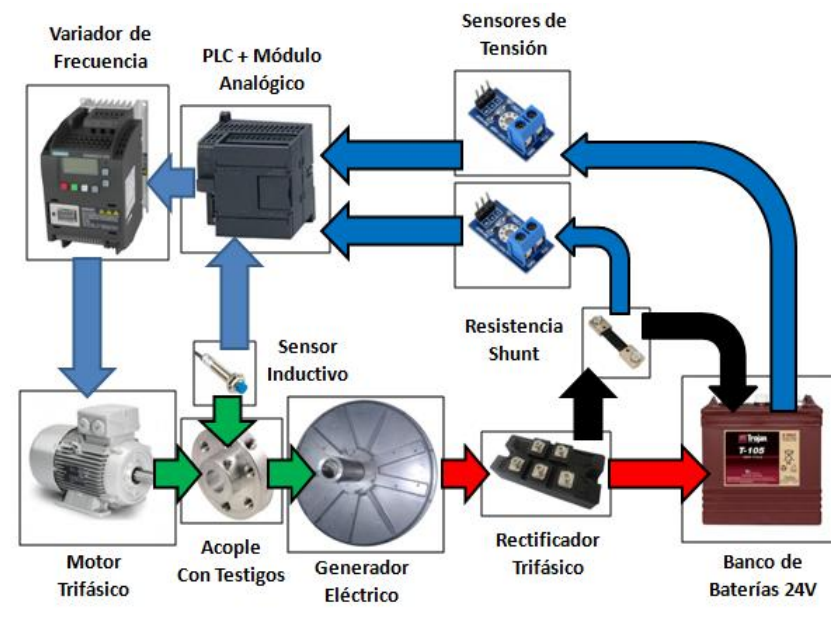

Fig 2. Esquema del banco de ensayos.

La CPU 222 dispone de 8 entradas digitales de 24 VDC y 6 salidas digitales de 24 VDC. EL módulo EM 235 [8]. dispone de 4 entradas analógicas y 1 salida analógica. Las entradas de este módulo son configurables a tensión y 
corriente en distintos rangos de funcionamiento. El variador de velocidad V20 dispone de 4 entradas digitales, 2 salidas digitales, 2 entradas analógicas y 1 salida analógica. Se utilizan las entradas digitales para habilitar el equipo, y la entrada analógica como consigna de velocidad.

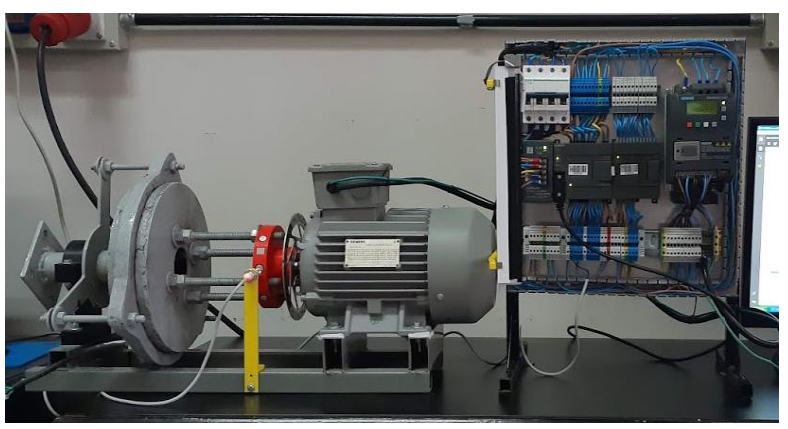

Fig 3. Banco de ensayos de generadores electricos

\section{B. Mediciones}

Los parámetros eléctricos que se midieron fueron la tensión y corriente a la salida del rectificador en continua, con lo cual se determinó la potencia eléctrica. De esta manera se espera realizar un balance energético y determinar el rendimiento del conjunto generador-rectificador.

La salida trifásica del generador de flujo axial depende directamente de la velocidad de rotación del mismo, influyendo en la amplitud de la tensión y corriente, y la frecuencia eléctrica. La señal alterna se rectifica mediante un puente trifásico compuesto por tres rectificadores para permitir la carga de las baterías. La corriente en continua que sale de los rectificadores se mide utilizando una resistencia shunt de precisión conectada en el cable negativo del circuito, y cuya caída de tensión se relaciona directamente con el valor de la corriente. Para la medición de tensión de la resistencia shunt y la del banco de baterías se utiliza el sensor de tensión FZ0430 compuesto por un divisor resistivo que permite medir tensiones de hasta $25 \mathrm{v}$ en corriente continua mediante una salida analógica de hasta $5 \mathrm{~V}$ relacionada linealmente con el valor de tensión medido. Las señales de ambos sensores se conectan con dos entradas del módulo analógico EM 235, las cuales se configuran para que lean señales de tensión en el rango de $0-5 \mathrm{~V}$ para permitir una resolución de $1,25 \mathrm{mV}$ [8]. En la programación del PLC, existen bloques específicos para el procesamiento de las señales analógicas, para relacionar el valor del conversor analógico-digital de la entrada analógica con la variable física leída por el sensor. En la figura 4 se observa los bloques de Normalizado "S_ITR" y Escalado "S_RTR", el primero permite convertir señales de entradas analógicas en valores normalizados entre 0.0 y 1.0 (tipo REAL), y el segundo convertir rangos de valores enteros de formato REAL (por ejemplo, los valores de entrada entre 0.0 y 1.0 a la zona de salida en porcentaje).
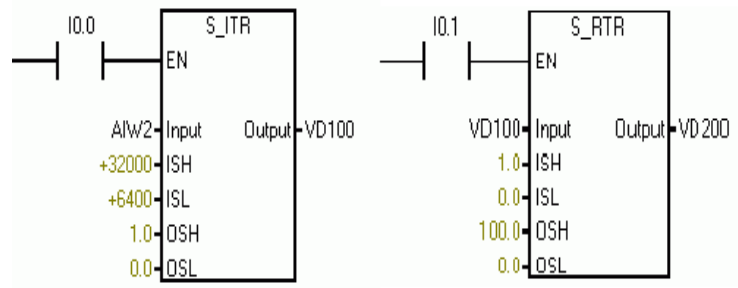

Fig 4. Banco de ensayos de generadores electricos.
El parámetro mecánico que se midió fue de velocidad de rotación (RPM). Se utilizó un sensor de tipo inductivo para realizar la lectura de RPM. La señal del sensor inductivo se realimenta hacia una entrada digital del PLC, esta es una señal pulsante con una frecuencia que está directamente relacionada con las RPM. Esta señal no puede ser procesada dentro del programa principal del PLC, dado que la señal pulsante de entrada tiene un periodo del orden de los milisegundos, y si este periodo es menor que el tiempo de ciclo del programa principal del PLC se perderán datos de entrada, es decir, la señal pulsante de entrada no podrá actualizarse en la imagen de proceso del PLC. Por esta razón se utilizó un contador rápido, cuya actualización no depende del tiempo de ciclo.

Existen dos formas de obtener el valor de RPM con un sensor inductivo y un PLC. Se puede determinar el tiempo que transcurre entre flancos ascendente (descendentes), es decir, el periodo de la señal, y de esta forma procesar ese valor aritméticamente para obtener el valor de RPM, o también se puede contabilizar la cantidad de pulsos en cierta base de tiempo (fija) y de esta forma procesar ese valor aritméticamente para obtener el valor de RPM. Estas dos estrategias tienen ventajas y desventajas. La primera tiene la ventaja de tener rápida actualización ante los cambios de RPM, su desventaja es que medir tiempos tan pequeños depende mucho del tipo de hardware que se dispone, en este caso la CPU 222 no admite este tipo de medición porque no dispone de los bloques correspondientes. La segunda forma tiene la ventaja de que solo se debe disponer de un contador rápido a nivel hardware, todos los controladores lógicos industriales disponen de esta función. Su principal desventaja es que el tiempo de respuesta depende del tiempo base establecido. Para el banco de ensayos se optó por la segunda forma para realizar la medición.

La medición primaria se realiza contando pulsos que el sensor inductivo realimenta al PLC, utilizando un contador rápido, configurado como se muestra en la figura 3. La serie S7 200 dispone de 6 tipos de HSC, que se pueden configurar en distintos modos. La diferencia entre los distintos modos de conteo está en la cantidad de fases, el control del sentido de contaje y el restablecimiento del contaje (interno o externo). Se utilizó una sola fase (sensor inductivo), el sentido de contaje y el restablecimiento se realiza externamente a través de una entrada digital.

Para programar el PLC se utiliza el software Step 7 Micro Win. En la figura 4 se observa la configuración, se utiliza el contador HSC0 en modo 4, con esta configuración la entrada digital I0.0 se utiliza como reloj, la entrada digital I0.1 se utiliza como control de sentido y la entrada digital I0.2 para el restablecimiento del contador.

En el programa principal se llama a la subrutina HSC_INT, la cual se configura solo una vez para no cargar al PLC con una acción repetitiva, la marca SM0.1 es la encargada de llamar a la subrutina solo en el primer ciclo del PLC. La primera línea de la subrutina mueve el valor hexadecimal 16\#F8 a la posición de memoria SMB37, configurando así la velocidad de conteo, el sentido del conteo y actualización al valor predeterminado de contaje. La posición de memoria SMD38 y SMD42 determina el valor actual y el valor predeterminado de HSC0 respectivamente, ambos se configuran a 0 . Además se determina que la configuración es para el HSC0 y que el modo 4.

El reinicio del valor del contador se realiza con un oscilador que activa la entrada digital I0.0 cada 1,5 segundos. 
El restablecimiento del contador se realiza con una salida digital Q0.4 que se realimenta hacia la entrada I0.0. Para que la actualización de esta salida no dependa del tiempo de ciclo utiliza una rutina de interrupción temporizada. Esta rutina se ejecuta en un intervalo de tiempo configurable cada $5 \mathrm{~ms}$. En la figura 6 se observa la configuración de esta subrutina.

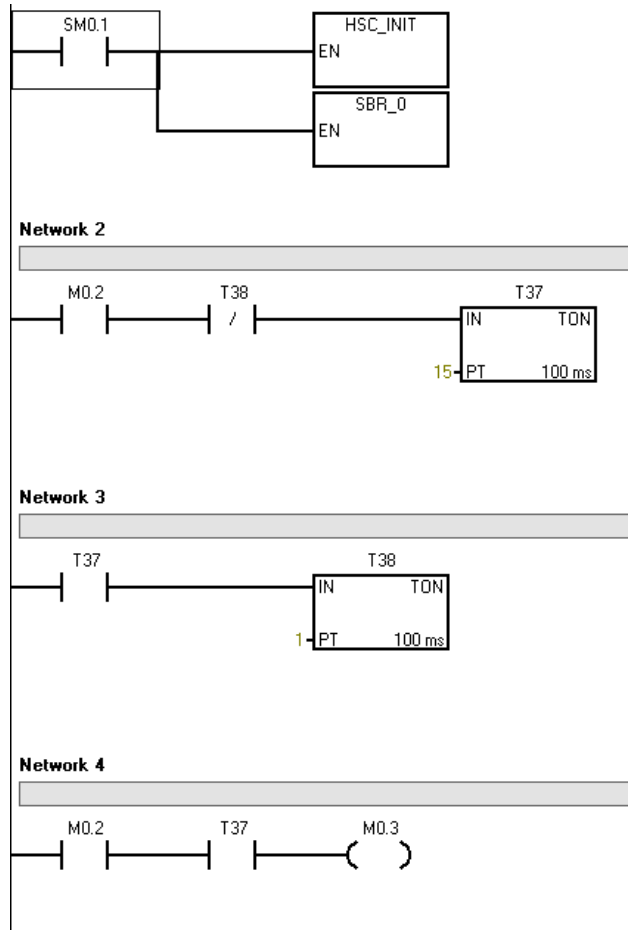

Fig 5. Configuración del contador rápido.

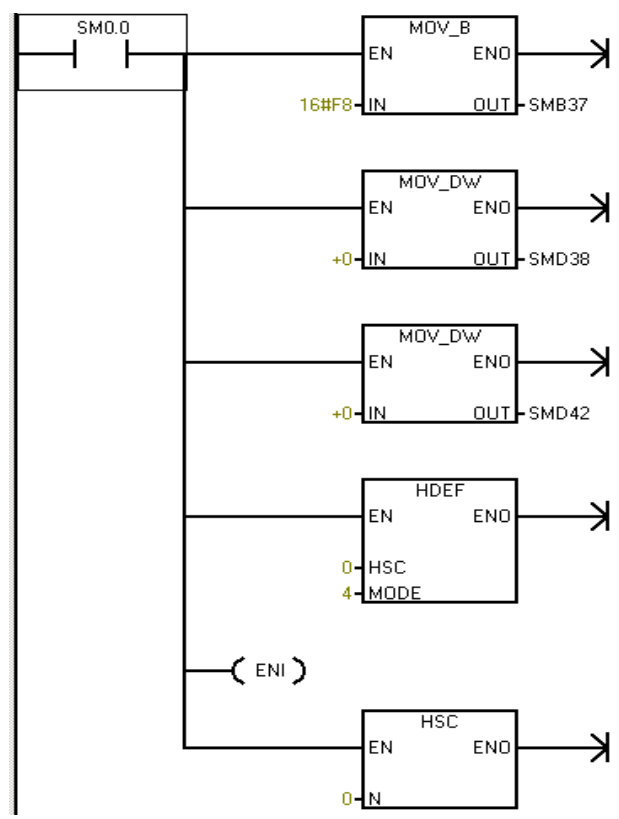

Fig 6. Subrutina de configuración de HSC0.

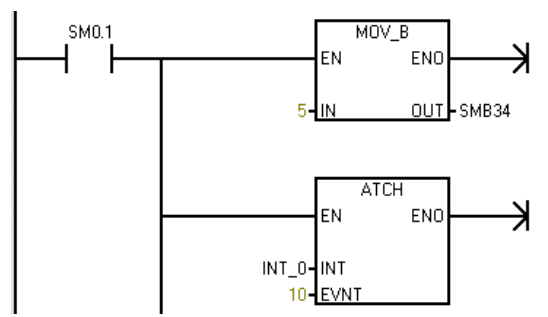

Fig 7. Configuración de la rutina de interrupción temporizada.

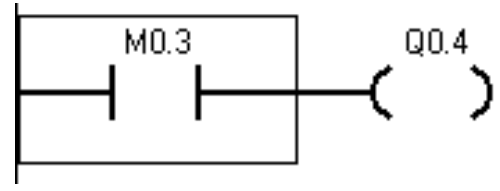

Fig 8. Interrupción temporizada.

El contador toma la cantidad de pulsos que el sensor inductivo realimenta por 1,5 segundos, el valor de conteo se ubica en la posición de memoria $\mathrm{HC} 0$ como un número entero. Para poder operar aritméticamente con este valor el programa lo convierte a un número real, a este valor lo multiplica por 60 segundos y lo divide entre la base de tiempo 1,5 segundos, por último este valor se divide entre la cantidad de pulsos por vuelta, y de esta forma se obtiene el valor de RPM.

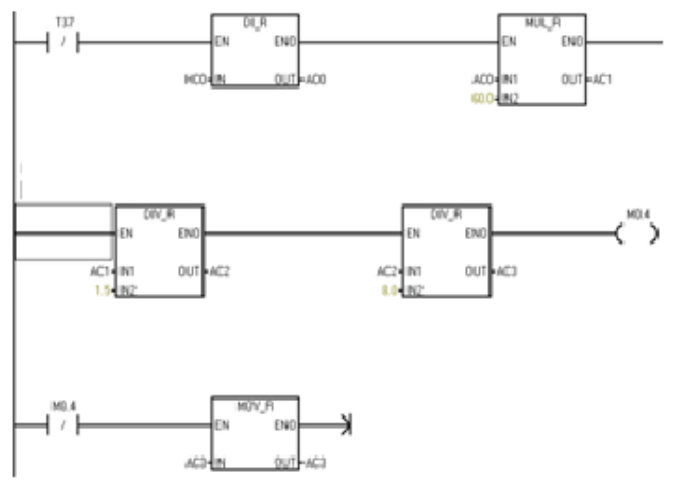

Fig 9. Aritmética para obtener las RPM.

Al momento de realizar la medición de RPM hay que tener en cuenta que el sensor inductivo detecta objetos ferromagnéticos, enviando una señal de $24 \mathrm{Vdc}$ a una entrada digital del PLC, que lo interpreta como un 1 lógico. Para la medición de RPM el sensor debe detectar, de alguna forma, el paso de un testigo de material ferromagnético. La cantidad de pulsos que el sensor detecta por vuelta está relacionada con la cantidad de testigos colocados en el eje que se desea medir las RPM. Se realizaron distintas pruebas para determinar la cantidad de pulsos por vuelta que se realimentarían al PLC. En principio se utilizó un pulso por vuelta, se observó que a altas RPM se tenía buena precisión, en cambio a bajas revoluciones (debajo de las 150 RPM) se tenía un error considerablemente alto. Cuando se aumentó el número de pulsos por vuelta, tanto a altas como a bajas RPM, se mejoró en precisión. Para el sistema de medición se optó por tener 8 pulsos por vuelta. Teóricamente se puede elevar el número de pulsos por vuelta tan grande para obtener gran precisión, sin embargo está limitado por la mecánica, es decir, por la forma física de obtener esta cantidad de pulsos.

En este proyecto se utilizó una impresora 3D para la fabricación de un acople con los testigos para realizar la medición de velocidad. En la figura 10 se observa el modelo final del acople que conecta el eje del motor con el rotor del generador. A esta pieza fabricada en impresora 3D, se le colocan en la periferia tornillos que serán los testigos para el sensor inductivo. De esta forma el sensor al detectar el paso de un testigo genera un pulso y por cada vuelta enviará 8 pulsos hacia el PLC. 


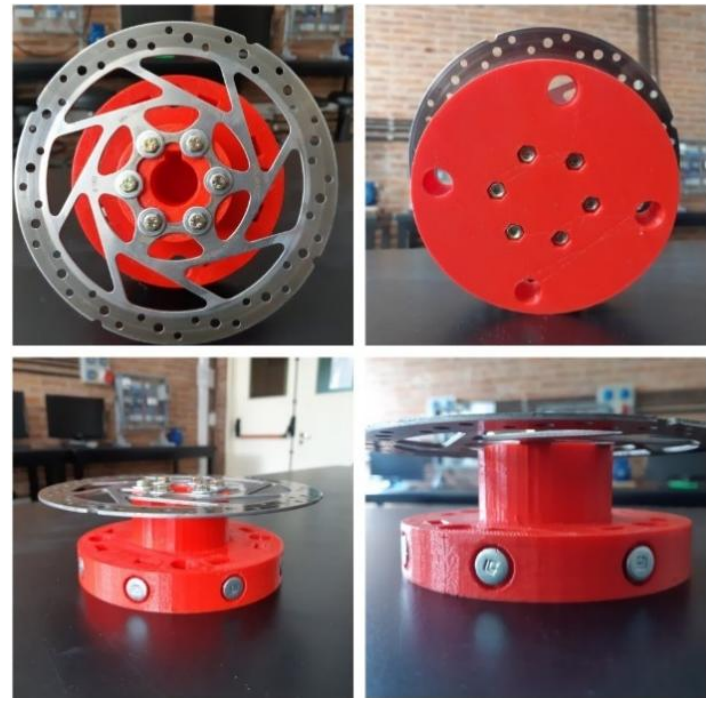

Fig 10. Acople mecánico entre el generador y el motor.

Para contrastar la medición de RPM del conjunto PLCsensor inductivo se utilizó un tacómetro digital, el cual permitió ver que tan precisa es la medición, y de esta forma definir la cantidad de pulsos por vuelta que debían realimentarse al PLC. En la figura 11 se observa el contraste entre la medición del sistema PLC - sensor inductivo (con 8 pulsos por vuelta) y el tacómetro digital. Se pueden distinguir dos zonas de medición. A bajas RPM el tacómetro digital tiene una mayor sensibilidad a los cambios de velocidad, diferentes es el caso para la medición con el sensor inductivo. A revoluciones por encima de las 10 RPM ambos sistemas de medición son aceptables.

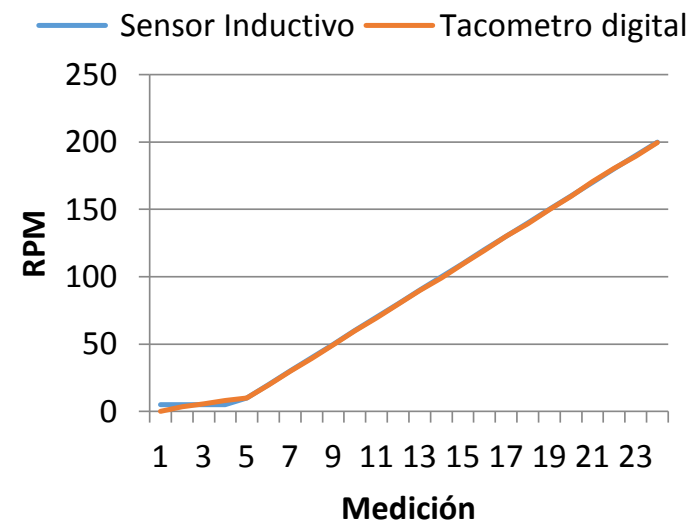

Fig 11. Comparación de mediciónes de RPM con tacómetro digital y con el sensor inductivo y PLC.

\section{Metodología del ensayo}

El ensayo del generador eléctrico considera el rango de RPM en el que funciona la turbina eólica Darrieus H, el cual comienza en 100 RPM aproximadamente, cuando la tensión rectificada del generador eléctrico supera los $24 \mathrm{~V}$ en continua, para que se produzca la carga de las baterías. El rango superior de funcionamiento se establece en 350 RPM.

Si la selección del modo es manual la velocidad del motor se establece a través de un potenciómetro en la entrada analógica del módulo EM235. Para analizar puntos intermedios, se realizan mediciones a 100, 150, 200, 250, 300 y 350 RPM, es decir, en cinco condiciones de funcionamiento para analizar el comportamiento mecánico y eléctrico.
Otro modo es la definición de rampas de RPM para establecer un funcionamiento con velocidades variables, y de esta forma simular las condiciones a las que el generador eléctrico estará sometido acoplado a la turbina eólica y obtener su respuesta de forma continua.

Se tiene proyectado otro tipo de ensayo en donde se buscará emular los vientos turbulentos que se dan en condiciones climáticas extremas y en sitios urbanos donde la superficie es rugosa. De esta forma se logrará poner al límite las condiciones eléctricas y mecánicas del generador, y obtener la respuesta lo más próxima a las que el generador estará sometido. La limitación que tiene este ensayo es que las RPM pueden cambiar en tiempos cortos, y el algoritmo de cálculo de RPM no pueda funcionar correctamente.

\section{RESULTADOS}

A partir de las mediciones realizadas sobre el banco de ensayos se construyeron los gráficos de la figura 12, 13 y 14, en las cuales se muestran la tensión y potencia en continua, respectivamente, en función de la velocidad del generador eléctrico.

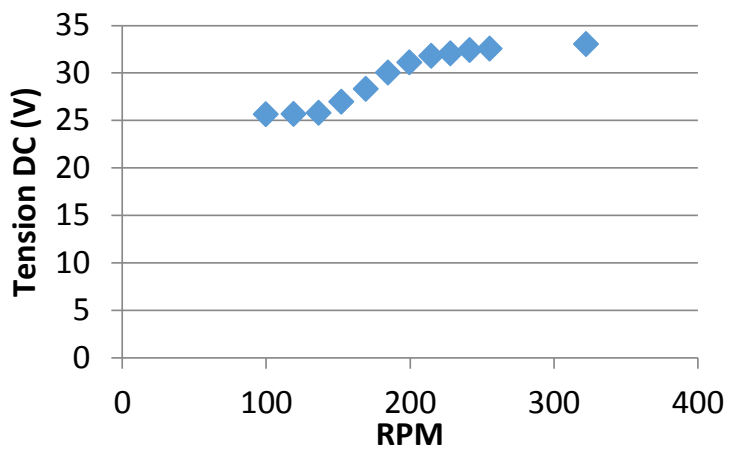

Fig 12. Curva de tensión en DC en función de las RPM.

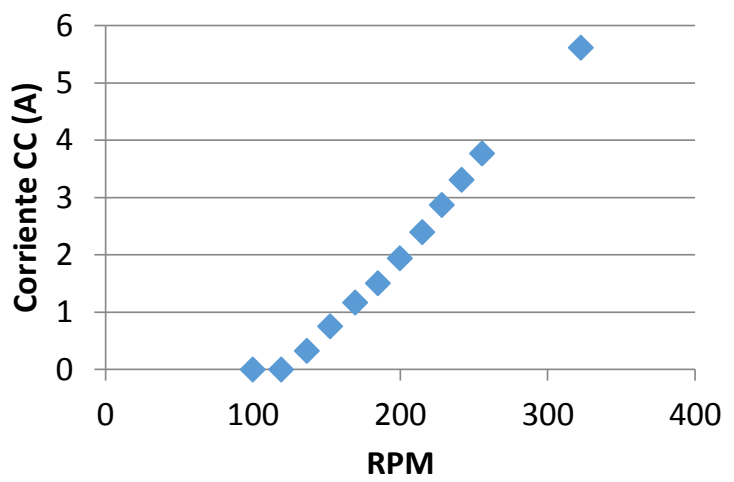

Fig 13. Curva de corriente en DC en función de las RPM.

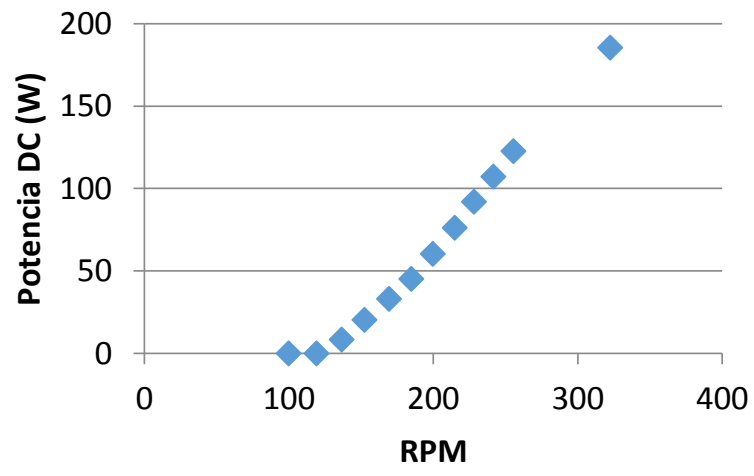

Fig 14. Curva de potencia en DC en funcion de las RPM. 
En el análisis de los gráficos se observa que el generador eléctrico comienza a entregar energía eléctrica al banco de baterías de $24 \mathrm{~V}$ a partir de 140 RPM, y crece de forma lineal con el aumento de la velocidad hasta alcanzar aproximadamente $180 \mathrm{~W}$ en continua a $300 \mathrm{RPM}$.

Estos resultados permiten obtener criterios para versiones futuras de aerogeneradores, tanto para el diseño de una nueva turbina, como también para el diseño de un generador eléctrico. Con respecto a la turbina, se parte de que existe el potencial eólico que puede ser aprovechado por el mismo generador eléctrico ensayado, es decir, que el punto de trabajo del generador eléctrico se puede mejorar, y de esta forma elevar la eficiencia del conjunto de la máquina. En cambio, cuando se habla de un nuevo diseño de generador eléctrico se hace hincapié en que el potencial eólico está fijo, y que se puede generar mayor energía a partir de cambio en el diseño de la máquina eléctrica, por ejemplo aumentando la cantidad de imanes permanentes, y de esta forma el número de polos de la máquina, consiguiendo que se genere potencia a menor velocidad de rotación de la turbina eólica, es decir, a menor velocidad de viento.

\section{ENSAYO DE MOTOR BRUSHLESS COMO GENERADOR}

En el marco del proyecto de investigación se está construyendo un generador eléctrico a partir de un motor brushless de $1 \mathrm{~kW}$, el cual se adaptará para ser instalado en un aerogenerador de eje vertical. En Europa y Estados Unidos es una modificación ampliamente utilizada en aerogeneradores de eje horizontal debido al bajo costo que implica tener un generador eléctrico a partir de pequeñas modificaciones mecánicas y eléctricas de un motor disponible en el mercado en distintas potencias y tamaños. En la figura 15 se observa el estator de 36 bobinas y el rotor de 12 imanes de ferrita que forman el motor brushless.

Para lograr que el motor funcione como generador, inicialmente, es necesario reconfigurar los bobinados del estator, ya que originalmente viene con una configuración trifásica de 12 bobinas por fase para ser alimentadas por 220 Vac con un convertidor electrónico. Sin embargo, como generador deberá conectarse a un banco de baterías de $24 \mathrm{Vdc}$. La reconexión de los bobinados en serie y paralelo se realizará para encontrar la configuración más apropiada para la velocidad de funcionamiento del aerogenerador. Por esta razón, será necesario estudiar y analizar el motor brushless como generador en el banco de ensayos y obtener las curvas de tensión de generación en vacío, tensión en carga, corriente y potencia en función de las RPM.

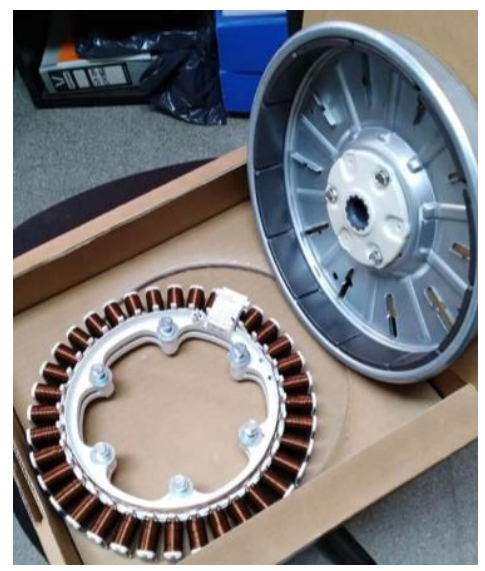

Fig 15. Estator y rotor del motor brushless
Para instalar el motor en el banco, se realizaron soportes específicos para acoplar el rotor de imanes de ferrita al motor trifásico y un soporte para vincular el estator con la estructura principal del banco, teniendo en cuenta el reducido entrehierro que hay entre las bobinas y los imanes. En la figura 16 se observa el motor brushless instalado en el banco de ensayos acoplado al motor trifásico.

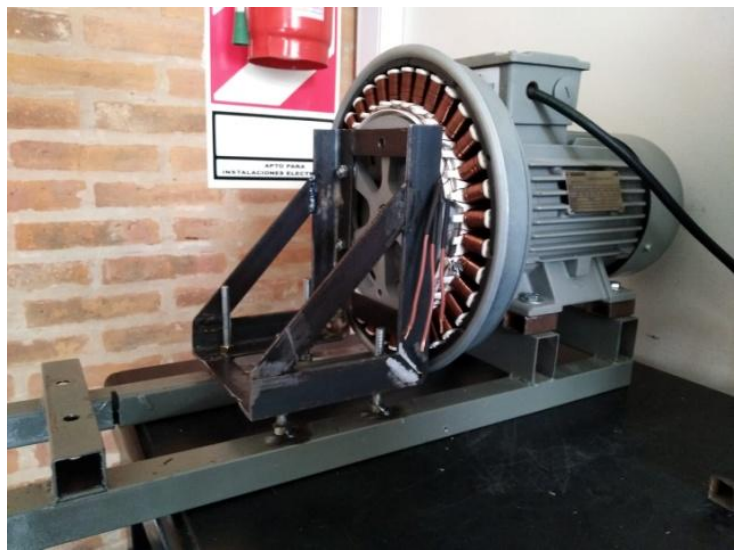

Fig 16. Instalación del motor brushless en el banco de ensayos.

\section{APLICACIONES EN DOCENCIA}

En la Universidad Nacional de General Sarmiento existe la carrera de Ingeniería Electromecánica y la Tecnicatura Superior en Automatización y Control, con lo cual, el banco de ensayos además de tener un uso como herramienta de investigación de generadores eléctricos, también se implementa en prácticas de asignaturas como Control de Máquinas Eléctricas e Instrumentación y Control. En las mismas, los estudiantes adquieren conocimientos sobre el manejo de motores eléctricos, variadores de velocidad e instrumentos industriales y los pueden aplicar directamente sobre el banco de ensayos.

\section{MEJORAS A FUTURO}

El banco de ensayos es una herramienta fundamental para el diseño y el ensayo de nuevos generadores eléctricos, con lo cual, se espera a futuro poder mejorarlo agregando sensores y dispositivos que permitan obtener mayor información durante un ensayo. La medición del torque que trasmite el motor trifásico al generador eléctrico, puede permitir junto con la medición de las RPM determinar la potencia mecánica que necesita el generador eléctrico y de esta manera, realizar un balance energético que permita conocer el rendimiento para distintos rangos de velocidad. Esta medición se realizará mediante un freno de Prony construido a partir de un sistema de frenado de bicicleta, con disco y zapata, el cual se acopla a una celda de carga para medir la fuerza que hace el sistema y en función del radio del disco de freno, obtener el torque. La celda de carga utiliza un conversor electrónico para adaptar la señal a la entrada analógica del PLC de 4 a $20 \mathrm{~mA}$. El diseño de una Interfaz HMI (Human Machine Interface) puede mejorar la realización de los ensayos y la visualización de la información obtenida por los sensores y los parámetros calculados por el PLC, independizándose de la necesidad de utilizar una PC, y permitiendo el almacenamiento de las mediciones y los cálculos en la misma memoria del equipo 
para ser extraída en cualquier momento a través de una memoria portátil.

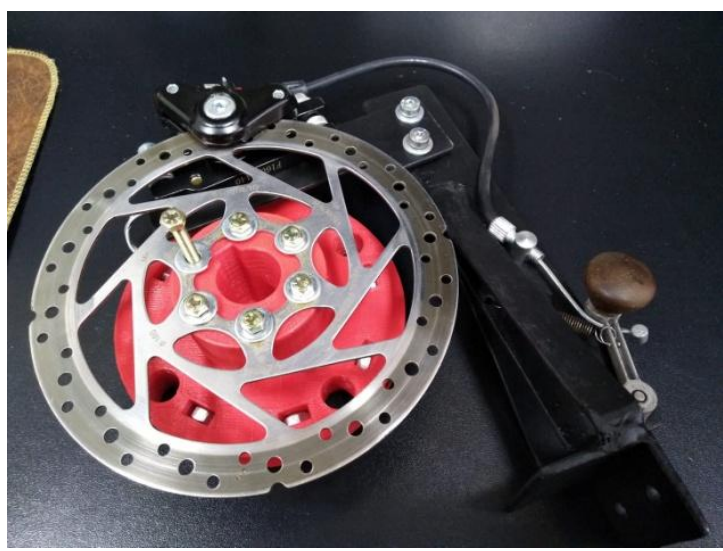

Fig 17. Construcción de Freno de Prony para medir torque.

\section{CONCLUSIONES}

A partir de la construcción de un banco de ensayos y la automatización del mismo, se obtuvo una herramienta para el estudio y el análisis de distintos tipos de generadores eléctricos. Inicialmente se pudo realizar la caracterización del funcionamiento de un generador eléctrico de flujo axial para ser implementado en un aerogenerador de baja potencia. Con los resultados del ensayo se pudo determinar cuál será la velocidad de arranque del generador eléctrico para producir energía y cuál será la velocidad máxima que soporte antes de que se activen los sistemas de protección del aerogenerador.
Actualmente se está realizando modificaciones sobre el banco para poder realizar los mismos ensayos en un generador eléctrico construido a partir de un motor brushless, el cual requiere la reconfiguración de sus bobinados para obtener potencia eléctrica deseada, para el rango de RPM en el que se espera que funcione.

\section{REFERENCIAS}

[1] Cammesa. (2019). Compania Administradora del Mercado Mayorista $\begin{array}{llll}\text { Eléctrico } & \text { S.A. } & \text { Retrieved }\end{array}$ https://despachorenovables.cammesa.com/renovables/

[2] Ahmed, E., \& Ahmed, S. (2018). Vertical Axis Wind Turbine. 10.13140/RG.2.2.12592.81927.

[3] Agotegaray, J. C., Pinzon Montes, A. L., \& Iratchet Prado, M. S. (2017). "Prototipo de aerogenerador de eje vertical Giromill". CLADI 2017. Congreso Latinoamericano de Ingenieria .

[4] Gourieres, D. L. (1983). Energía Eólica. Teoría, concepción y cálculo práctico de las instalaciones. Masson S.A.

[5] Piggot, Hugh (2003). How to build a wind generator - the axial flux alternator. Link: https://www.scoraigwind.com/pirate/Hugh Piggott Axial-flow PMG wind turbine May 2003.pdf

[6] Siemens. (2012, Febrero). SIMOTICS Low-voltage Motors. E86060K5581-A111-A4-7600.

[7] AG, S. (2013, Febrero ). "Convetidor SINAMICS V20". A5E31842763.

[8] AG, S. (2008, Agosto). "Manual del sistema de automatizacion S7200". 6ES7298--8FA24--8DH0.

[9] Latoufis, K.C., Messinis, G.M., Kotsampopoulos P.C \& Hatziargyriou N.D. (2012). Axial flux permanent magnet generator design for low cost manufacturing of small wind turbinesGenerador axial. Wind Engineering Volume 36, no. 4, 2012 pp 411-442 